\title{
Effect of COVID-19 Non-Pharmaceutical Interventions and Threats to Human Rights
}

Ha Hwang ( $\square$ ghkdgk0309@gmail.com )

Korea Institute of Public Administration https://orcid.org/0000-0001-8983-0139

\section{Seung-Hun Hong}

Korea Institute of Public Administration

\section{Min-Hye Park}

Ulsan National Institute of Science and Technology

\section{Biological Sciences - Article}

Keywords: COVID-19, non-pharmaceutical interventions (NPIs), human rights

Posted Date: August 26th, 2020

DOl: https://doi.org/10.21203/rs.3.rs-64304/v1

License: (c) (i) This work is licensed under a Creative Commons Attribution 4.0 International License. Read Full License 


\section{Introduction}

In his eloquent explanation of Panopticism, Foucault described how a $17^{\text {th }}$ Century European State hit by plague could shut down local communities and put surveillance on every corner of the street to oppress people's movement and assembly ${ }^{1}$. What was happening in several liberal democracies four centuries later was not so much different. When faced with another uncontrollable epidemic, governments decided swiftly to order regional or national lockdown based on rare evidence that such harsh measures were effective. Indeed, the COVID-19 epidemic had proceeded to a global pandemic with unprecedented pace and scope, allowing governments little time to adopt responses that protect citizens' life and observe their human rights. A growing concern is that such interventions, aimed at ensuring public safety, may severely violate fundamental human rights ${ }^{2}$. Given that public safety is also a kind of human rights essential to a democratic society, it is vital to explore the extent to which State emergency power is exercised to contain the spread of COVID-19 effectively while minimizing the risk of human rights abuse.

The burgeoning literature examines the effect of non-pharmaceutical interventions (NPIs) ${ }^{3-7}$, the stringency of $\mathrm{NPIs}^{8}$, or factors affecting compliance with NPIs ${ }^{9}$. Some mainly support the effect of lockdown by arguing that stringent government responses are effective in containing COVID19 , but only in a single country ${ }^{3,6}$, or even without giving what it means by lockdown in different countries $^{5,10,11}$. Given the variety of scope and measures included in a lockdown of different countries, however, it is crucial to investigate the effect of NPIs in a more detailed way. The Oxford COVID-19 Government Response Tracker (OxCGRT) database ${ }^{8}$, which tracks down twelve COVID-19 government responses over 178 countries, indicates that only four countries have implemented complete lockdown, a combination of all assembly and movement restrictions.

This paper examines which NPIs are more effective than others in containing COVID-19 with the consideration of their threat to human rights. Two tasks are tackled using publicly available data developed by OxCGRT: First, we examine the effectiveness of NPIs in containing COVID19 over 108 countries for the period from the first day of January until the 15th of June 2020, when some countries began to relax the intervention. For a more nuanced analysis, we classify NPIs into three categories according to their threat to human rights: the right to freedom of assembly, the right to freedom of movement, and the right to privacy. This categorization enables us to assess the variation of government approaches to containing COVID-19 and evaluate the effect of such approaches on curbing the transmission. Second, we select a total of ten countries - five from North America and Europe, and another five from Asia and the Pacific - to visualize the cumulative number of confirmed cases and the change in the NPI levels over time. This provides supporting evidence of the regression analyses that shows the change of individual countries' responding strategies and their effects as the confirmed cases increase. 
This paper finds that school closure is effective in containing COVID-19 only when it is implemented along with complete contact tracing. All other NPIs are neither statistically significant nor positively associated with the deterrence of COVID-19. Our findings imply that, to contain COVID-19 effectively and minimize the risk of human rights violations, governments should consider implementing prudently designed full contact tracing and school closure policies, among others. Other interventions limiting freedom of movement and assembly should be carefully adopted with minimal infringement of human rights.

\section{NPIs and consideration for human rights}

The range of human rights at risk during the COVID-19 pandemic is extensive: from rights to life, health, and education to the rights to freedom of movement, public assembly, expression, and many more. This paper does not purport to address all those issues. It instead focuses on three types of human rights that are presumably under direct threat by the implementation of NPIs. We selected nine responses in OxCGRT and broke them down into three categories according to human rights such responses may restrain.

One purpose of NPIs is to limit people's movement, which inevitably leads to interfering with the freedom to move. Stay at home requirements, for example, are intended to get both infected and uninfected people not to move around so that they stay away from potential virus transmission. NPIs interfering with freedom of movement include public transport closure, domestic travel restriction, and international travel ban, which are all intended to mitigate, among others, the risk of virus transmission caused by human movement. Some studies assume that NPIs are purported only to restrain mobility ${ }^{12}$.

However, NPIs may also put restrictions on human gathering and thus restrain freedom of assembly. This type of NPIs aims at mitigating the risk of virus transmission caused by people's gathering in a place rather than transmission caused by people's moving from one location to another. NPIs of this sort include school closure, workplace closure, public event cancellation, and gathering size restrictions. More serious human rights concerns may be associated with contact tracing - that is, to track down personal contacts of all (or selected) confirmed cases. The goal of contact tracing is to trace virus transmission via direct human contact to find out those who are exposed to virus infection. However, its side effects remain that tracking personal contacts may give rise to too much State surveillance over the private sphere. Several countries, for example, were less inclined to adopt full contact tracing policies due to rising concerns over privacy violation ${ }^{13,14}$. $<$ Table $1>$ summarizes the categorization of NPIs used in this study.

$<$ Table 1 $>$ Summary of NPIs considered

\begin{tabular}{llll}
\hline Classification & Label & NPIs & Description 1 (Degree) \\
\cline { 4 - 4 } & & Description 2 (Targeted or General) \\
\hline $\begin{array}{l}\text { Assembly } \\
\text { restriction }\end{array}$ & A1 & School closing & $\begin{array}{l}\text { 0 - No measures; 1 - recommend closing; 2 - Require closing (only some } \\
\text { levels or categories, e.g., just high school, or just public schools); and 3 - }\end{array}$ \\
\hline
\end{tabular}


Require closing all levels

0 - Targeted; and 1 - General

\begin{tabular}{|c|c|c|}
\hline & & 0 - Targeted; and 1 - General \\
\hline \multirow[t]{2}{*}{ A2 } & \multirow[t]{2}{*}{ Workplace closing } & $\begin{array}{l}0 \text { - No measures; } 1 \text { - recommend closing (or work from home); } 2 \text { - require } \\
\text { closing (or work from home) for some sectors or categories of workers; } \\
\text { and } 3 \text { - require closing (or work from home) all-but-essential workplaces } \\
\text { (e.g., grocery stores, doctors) }\end{array}$ \\
\hline & & 0 - Targeted; and 1 - General \\
\hline
\end{tabular}

\begin{tabular}{|c|c|c|}
\hline \multirow[t]{2}{*}{ A3 } & \multirow[t]{2}{*}{ Cancel public events } & 0- No measures; 1 - Recommend canceling; and 2 - Require canceling \\
\hline & & 0 - Targeted; and 1 - General \\
\hline \multirow[t]{2}{*}{ A4 } & \multirow[t]{2}{*}{$\begin{array}{l}\text { Restrictions on } \\
\text { gathering size }\end{array}$} & $\begin{array}{l}0 \text { - No restrictions; } 1 \text { - Restrictions on very large gatherings (the limit is } \\
\text { above } 1000 \text { people); } 2 \text { - Restrictions on gatherings between } 101-1000 \\
\text { people; } 3 \text { - Restrictions on gatherings between } 11-100 \text { people; and } 4 \text { - } \\
\text { Restrictions on gatherings of } 10 \text { people or less }\end{array}$ \\
\hline & & 0 - Targeted; and 1 - General \\
\hline
\end{tabular}

Movement $\quad$ M1 Close public transport $\quad 0$ - No measures; 1 - Recommend closing (or significantly reduce

restriction volume/route/means of transport available); and 2 - Require closing (or prohibit most citizens from using it)

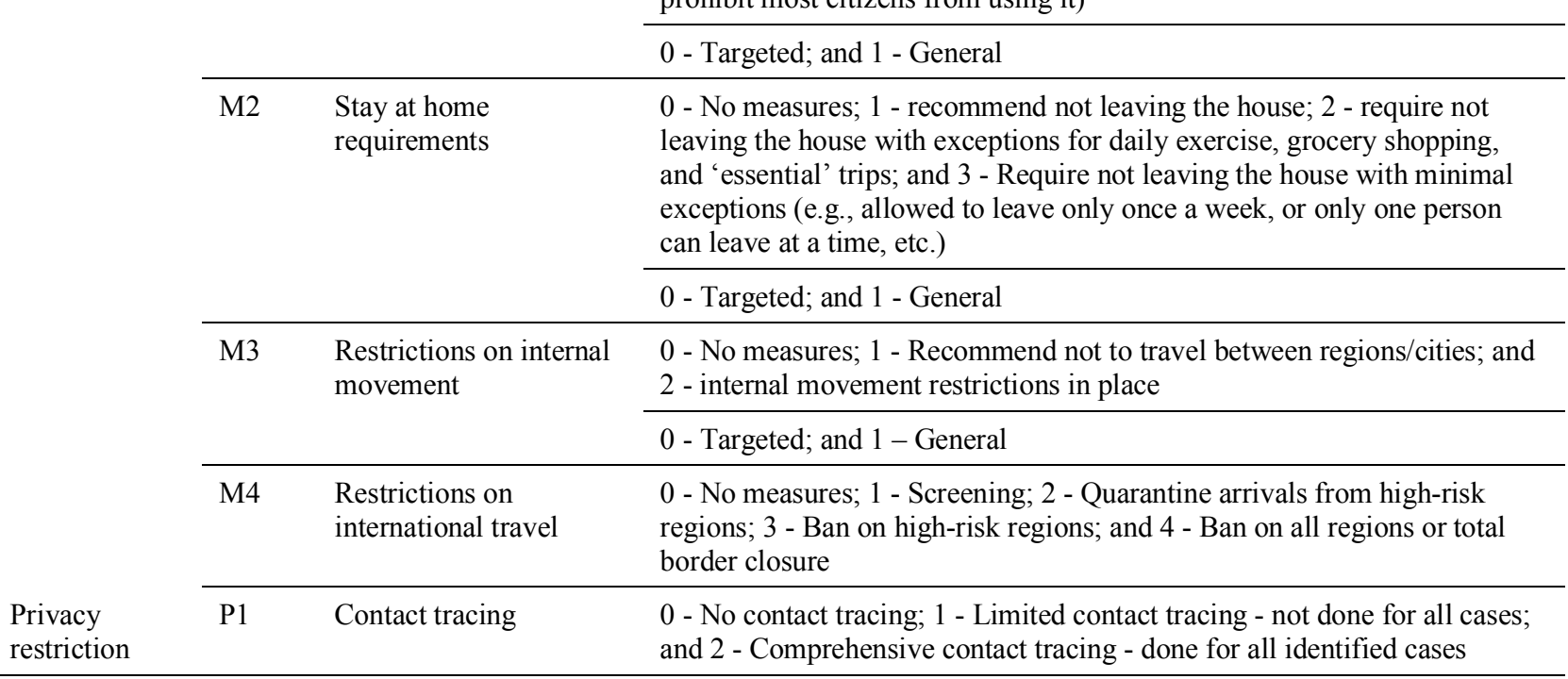

Source: OxCGRT ${ }^{8}$

\section{Results}

Decrease rate of increase in the cumulative number of COVID-19 cases

Some previous studies used the number of daily confirmed cases or deaths as explained variables to identify the effect of NPIs on containing COVID $19^{4-8}$. However, daily confirmed cases or deaths are not suitable for quantitatively estimating the effectiveness of NPIs because the daily deviation is large, and the effects do not appear immediately after implementing these policies due to the incubation period of the virus. As an alternative, we calculated a decrease rate of increase in the cumulated number of confirmed cases (DRICs) of COVID-19 (see Methods). To generate this variable, the highest value of the average increase rate of the cumulated number of confirmed cases (IRCs) between the $1^{\text {st }}$ of January and the $15^{\text {th }}$ of June 2020 was divided by the average IRCs six days after the record date for each country, considering the incubation period ${ }^{4}$. 
The more the IRCs decreases during the incubation period, the greater the value of DRICs. We used this value as the dependent variable to estimate the effect of NPIs applied at the time when the IRCs was highest. Of course, there are many cases where the dates the highest IRCs were recorded and the dates associated NPIs were implemented do not coincide. Considering that the incubation period of COVID-19 is about six days, however, it is an appropriate assumption. A total of 108 countries were included in the linear regression analyses except for countries in which the cumulated number of confirmed case graphs do not show a sigmoid curve or calculation of DRICs is impossible due to data missing or error. The average value of DRICs is about 1.599, which indicates that the countries used in the analysis showed a decrease in IRCs to the $62.5 \%(1 / 1.599)$ level on average after six days when the IRCs were recorded highest.

\section{Effect of NPIs to contain COVID-19}

We established linear regression models with DRICs as the dependent variable and NPIs as the independent variables to confirm the effect of NPIs on containing COVID-19. The standardization of NPIs relies on the method suggested by OxCGRT ${ }^{8}$. One difference is that the scale of the variables is normalized from 0 to 1 . $<$ Table $1>$ shows details of the explanatory variables, and $<$ Table $2>$ displays descriptive statistics. School closing is the most widely and strongly implemented NPI and cancel public events is the second.

$<$ Table $2>$ Descriptive statistics of the regression variables

\begin{tabular}{lllllll}
\hline Variables & Mean & Median & SD & Max & Min & N \\
\hline School closing (A1) & 0.931 & 1.000 & 0.193 & 1.000 & 0.000 & 108 \\
\hline Workplace closing (A2) & 0.671 & 0.667 & 0.322 & 1.000 & 0.000 & 108 \\
\hline Cancel public events (A3) & 0.904 & 1.000 & 0.231 & 1.000 & 0.000 & 108 \\
\hline Gathering size restriction (A4) & 0.729 & 0.875 & 0.359 & 1.000 & 0.000 & 108 \\
\hline Close public transport (M1) & 0.463 & 0.500 & 0.376 & 1.000 & 0.000 & 108 \\
\hline Stay at home requirement (M2) & 0.457 & 0.500 & 0.290 & 1.000 & 0.000 & 108 \\
\hline Internal movement restrictions (M3) & 0.631 & 0.750 & 0.380 & 1.000 & 0.000 & 108 \\
\hline International travel restrictions (M4) & 0.855 & 1.000 & 0.258 & 1.000 & 0.000 & 108 \\
\hline Contact tracing (P1) & 0.638 & 0.500 & 0.353 & 1.000 & 0.000 & 108 \\
\hline
\end{tabular}

The correlation matrix of the dependent and independent variables used in the regression models shows that only school closing and contact tracing interventions are positively correlated with DRICs while others show negative correlations (see $<$ Table $3>$ ). Among independent variables, stay at home requirements and internal movement restrictions are highly correlated $(\mathrm{r}=0.71)$, and 
other variables show moderate or low correlations. However, the highest score of the variance inflation factor (VIF) is 2.42, which suggests very weak evidence of multicollinearity.

$<$ Table $3>$ Correlation analysis of the regression variables

\begin{tabular}{|c|c|c|c|c|c|c|c|c|c|c|c|}
\hline & DRICs & A1 & $\mathrm{A} 2$ & A3 & A4 & M1 & M2 & M3 & M4 & P1 & VIF \\
\hline DRICs & 1.00 & & & & & & & & & & - \\
\hline A1 & 0.02 & 1.00 & & & & & & & & & 1.55 \\
\hline A2 & -0.24 & 0.39 & 1.00 & & & & & & & & 1.67 \\
\hline A3 & -0.35 & 0.58 & 0.37 & 1.00 & & & & & & & 1.88 \\
\hline A4 & -0.13 & 0.37 & 0.48 & 0.39 & 1.00 & & & & & & 1.39 \\
\hline M1 & -0.11 & 0.36 & 0.44 & 0.37 & 0.38 & 1.00 & & & & & 1.41 \\
\hline M2 & -0.26 & 0.41 & 0.58 & 0.47 & 0.43 & 0.49 & 1.00 & & & & 2.42 \\
\hline M3 & -0.32 & 0.38 & 0.45 & 0.44 & 0.41 & 0.49 & 0.71 & 1.00 & & & 2.10 \\
\hline M4 & -0.21 & 0.10 & -0.02 & 0.36 & 0.19 & 0.17 & 0.09 & 0.17 & 1.00 & & 1.17 \\
\hline P1 & 0.24 & -0.05 & -0.10 & -0.16 & -0.07 & -0.09 & -0.07 & -0.15 & 0.04 & 1.00 & 1.02 \\
\hline
\end{tabular}

$<$ Table $4>$ displays the results of the linear regression models. According to the results of Model 1, school closure has a strong, positive impact on containing COVID-19, while the rest of NPIs is statistically insignificant or has negative effects. These conflicting results may draw on the unpredictability of people's behaviors when their freedoms of movement and assembly are limited. When restricting people's gathering or movement, a government expects them to stay in their own homes. The negative correlation between most NPIs except school closure and DRICs implies that such measure, when implemented individually, would rather increase other communal activities. A study using Google's mobility data reveals that people's mobility has diversified since the outbreak of COVID-19 rather than having been restrained ${ }^{12}$.

On the contrary, school closure is effective because, even though schools are closed, school terms still carry on and students are obliged to fulfill their distant learning at home. At school, students would have to stay in the same and closed place for hours keeping intimate contact with each other. Besides, as younger students stay at home to take online classes, at least one parent would have to look after them, which would result in fewer outdoor activities and more compliance with stay at home policies. 
$<$ Table $4>$ Regression results by models

\begin{tabular}{rrrr}
\hline Variable & Model 1 $(\mathrm{n}=108)$ & Model 2 $(\mathrm{n}=108)$ & Model 3 (n=108) \\
\hline Intercept & $2.063(0.336)^{* * *}$ & $1.813(0.833)^{*}$ & $1.467(0.884)$ \\
School closing (A1) & $1.328(0.382)^{* * *}$ & $-2.070(1.010)^{*}$ & $-1.887(1.052) \cdot$ \\
Workplace closing (A2) & $-0.434(0.239) \cdot$ & $0.434(0.459)$ & $0.866(0.514)^{*}$ \\
Cancel public events (A3) & $-1.226(0.349)^{* * *}$ & $1.568(0.995)$ & $1.625(1.004)$ \\
Gathering size restrictions (A4) & $0.136(0.195)$ & $0.194(0.367)$ & $0.178(0.393)$ \\
Close public transport (M1) & $0.204(0.189)$ & $0.209(0.177)$ & $-0.202(0.398)$ \\
Stay at home requirements (M2) & $0.055(0.318)$ & $0.051(0.295)$ & $-0.823(0.650)$ \\
Internal movement restrictions (M3) & $-0.498(0.227)^{*}$ & $-0.445(0.212)^{*}$ & $-0.170(0.445)$ \\
International travel restrictions (M4) & $-0.238(0.248)$ & $-0.308(0.235)$ & $0.024(0.418)$ \\
\hline Contact tracing (P1) & & $0.227(1.033)$ & $0.774(1.159)$ \\
A1:P1 & & $4.162(1.216)^{* * *}$ & $3.930(1.283)^{* *}$ \\
A2:P1 & & $-1.221(0.610)^{*}$ & $-1.771(0.678)^{*}$ \\
A3:P1 & & $-3.163(1.128)^{* *}$ & $-3.245(1.156)^{* *}$ \\
A4:P1 & & $-0.053(0.556)$ & $-0.093(0.594)$ \\
\hline M1:P1 & & & $0.627(0.534)$ \\
M2:P1 & & & $1.176(0.832)$ \\
M3:P1 & 0.228 & & $-0.436(0.595)$ \\
M4:P1 & & 0.343 & $-0.520(0.605)$ \\
\hline Adjusted R-squared & & 0.346 \\
\hline N & & &
\end{tabular}

Note: $* * * P<0.001,{ }^{* *} P<0.01, * P<0.05, \cdot P<0.1$

Model 2 and 3 show the effects of the sequential combination of contact tracing, a privacyrelated variable, with variables related to assembly restriction and movement restriction. Model 2, which includes variables that combine contact tracing and assembly restriction variables, shows an $11.5 \%$ increase in explanatory power (Adj. R-squared=0.343) than Model 1 (Adj. Rsquared $=0.228$, and it turns out that 3 out of 4 combined variables are statistically significant. On the other hand, Model 3 shows only a $0.3 \%$ increase in explanatory power (Adj. Rsquared $=0.346$ ) compared to Model 2 , and there was no additional variable that shows statistical significance.

According to the results of Model 2, the effect of school closure is $(-2.070+4.162 * \mathrm{P} 1)$. Therefore, the complete contact tracing policy $(\mathrm{P} 1=1)$ must be applied together to have the effect 
of containing COVID-19 through school closure. The effect of contact tracing is $(4.162 * \mathrm{~A} 1$ $1.221 * \mathrm{~A} 2-3.163 * \mathrm{~A} 3)$. Unlike school closure, workplace closure and canceling public events appear to reduce the effectiveness of contact tracing. One reason may be 2 that limiting predictable gatherings may push people to take unpredictable gathering and movement behaviors rather than having them stay at home ${ }^{3}$.

We found that school closure is effective in containing COVID-19 only when it is implemented along with complete contact tracing. Then, our next question was whether there is a clear difference in the degree of containing COVID-19 between countries adopting limited contact tracing and those implementing complete contact tracing. DRICs scores of countries with limited contact tracing $(n=47)$ and those with complete contact tracing $(n=46)$ are 1.372 and 1.795, respectively, and the difference between the two scores is statistically significant $(\mathrm{t}=-3.166, \mathrm{df}=$ 54.031, p-value $=0.003$ ). The IRCs of countries with limited contact tracing decreased to about $72.9 \%$ (1/1.372) after six days from the highest, while those with complete contact tracing decreased to $55.7 \%(1 / 1.795)$. This result shows that stronger contact tracing policy is more effective in containing COVID-19.

The results of our linear regression analysis are meaningful in that they correlate the effect of governments' NPIs by country in the period when confirmed cases increase most. Still, there are limitations in showing the change of individual countries' response strategies and their effects. Therefore, we confirm the results of the regression analysis by examining the changes in the cumulated number of confirmed cases and the changes in government policies of ten selected countries. Five countries are selected from North America and Europe, and another five from Asia and the Pacific (see Figure1). To present more explicit graphs, we combine NPIs for assembly restrictions and movement restrictions into one indicator, each using the method suggested by $\mathrm{OxCGRT}^{8}$ (see Methods). 
a. United Kingdom

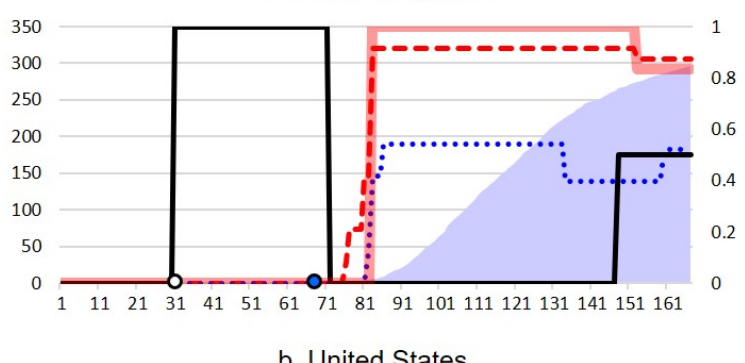

b. United States

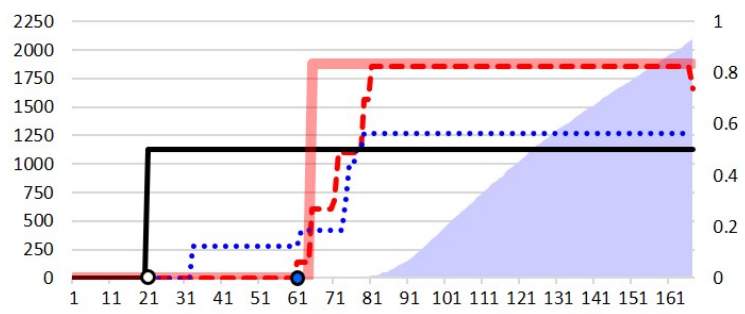

c. Sweden

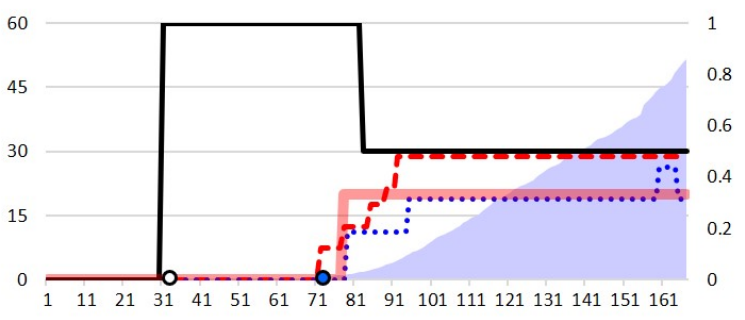

d. France

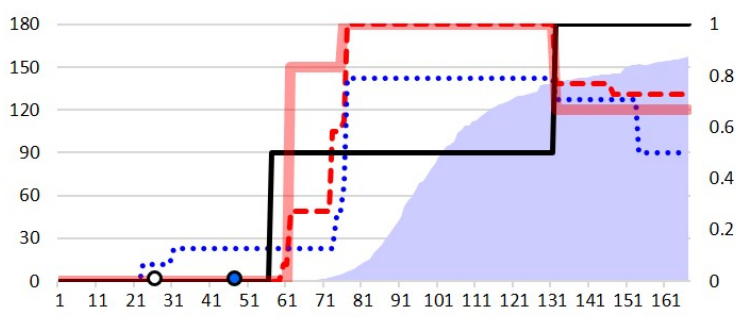

e. Slovenia

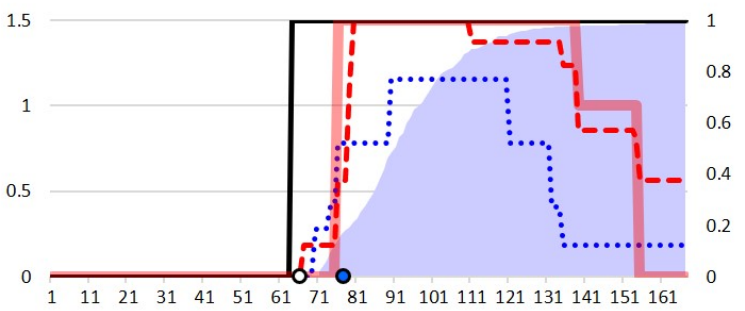

Cumulative number of confirmed cases

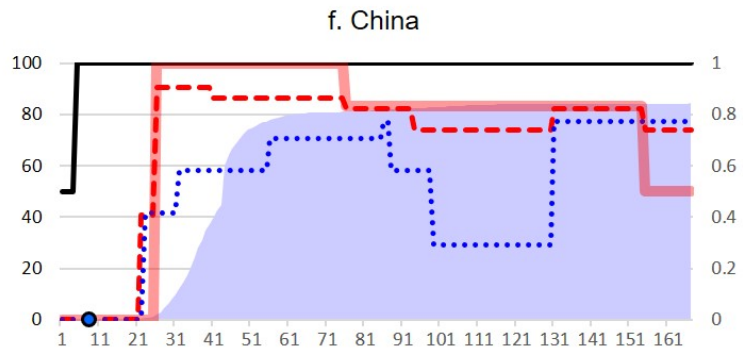

g. South Korea

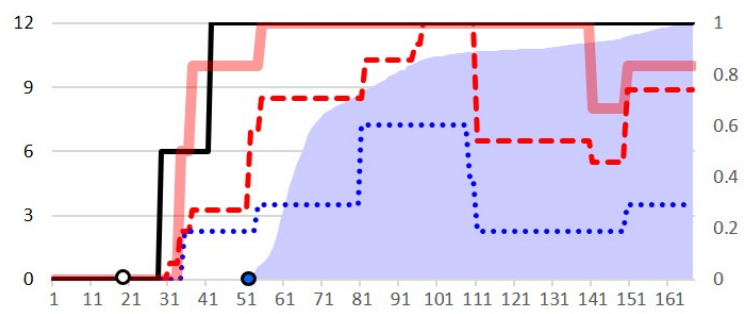

h. Japan

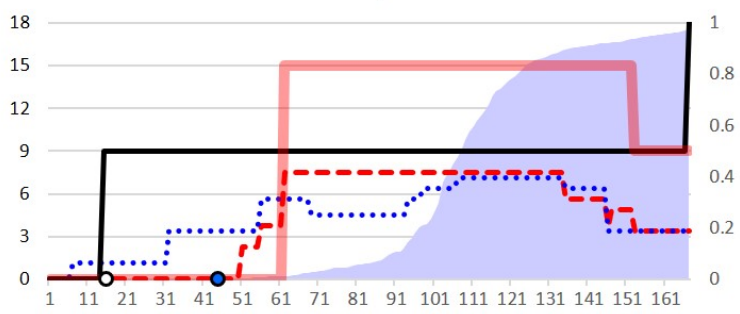

i. Vietnam

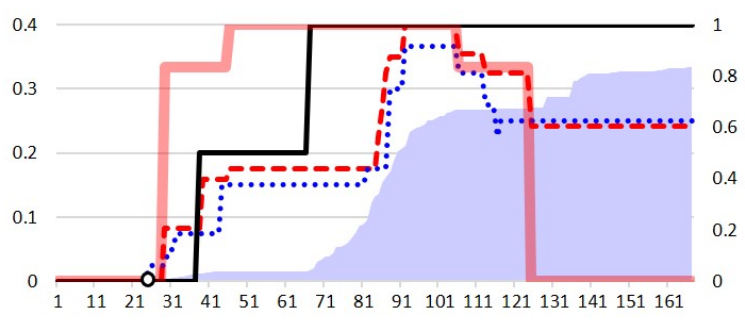

j. New Zealand

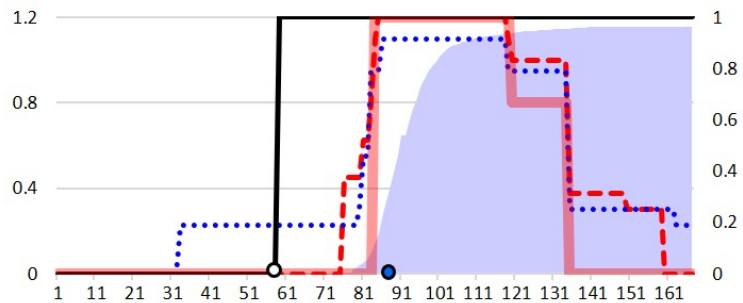

- $1^{\text {st }}$ confirmed case

- $1^{\text {st }}$ death

Movement restriction

Privacy restriction

$<$ Figure 1> Changes in NPIs and cumulated number of confirmed cases of COVID-19 of the selected countries Note: The horizontal axis denotes the number of days from the $1^{\text {st }}$ of January, 2020. The right vertical axis denotes the standardized score of NPIs. The left vertical axis denotes the number of confirmed cases of COVID-19, and the unit of confirmed cases is a thousand people. 
The graphs demonstrate that in most countries, the stringency of assembly and movement restrictions surges in response to the increase of newly confirmed cases. A striking difference is that countries which implemented complete contact tracing from a very early stage of the COVID-19 epidemic, such as China, South Korea, Vietnam, Slovenia, and New Zealand, saturate the cumulated number of confirmed case curve rapidly, while this is not the case for countries which did not implement full contact tracing. Also, when the intensive school closure and the complete contact tracing were implemented at the same time, IRCs has rapidly eased.

Slovenia and New Zealand are typical examples of success: both countries began to implement complete contact tracing before the number of confirmed cases surged, adopted other NPIs in proportion to the increase of confirmed cases, and relaxed assembly and movement restrictions, while keeping full contact tracing, when the increase became stabilized. South Korea can be another example of success: implementing full contact tracing throughout the outbreak and maneuvering the stringency of movement and assembly restrictions in response to the number of daily confirmed cases. These approaches were useful not only in containing COVID-19 but also in minimizing government interventions on citizens' human rights. On the contrary, the number of confirmed cases continued to grow in the UK, US, and Sweden, where contact tracing was partially implemented or not even implemented for a long time after the $1^{\text {st }}$ confirmed case. Gradually strengthening assembly and movement restrictions, which was not associated with complete contact tracing, could not help deter the virus transmission in these countries.

\section{Extending findings}

Our findings show that school closure must be accompanied by complete contact tracing if it gets to be effective in containing COVID-19. All other NPIs are proven to be statistically insignificant or even have a negative impact. Three major arguments can be drawn. First, when it comes to design government responses to contain an epidemic that restrains people's human rights, a government is required to realize that people's desire for freedom cannot be easily controlled. The negative impacts of workplace closure, public event cancelation, and gathering size restrictions show that restraining one form of gatherings may push people to take other unforeseeable modes of gathering or mobility, and consequentially, make it even harder for governments to trace people's contacts.

Second, full contact tracing needs to be followed up by at least two measures: comprehensive testing of traced people and treatment of confirmed cases. Considering that around one-third of confirmed cases is asymptomatic ${ }^{15,16}$, it is especially important to test all contacts of confirmed people to figure out who was infected and who was not. And then, those confirmed people need to be isolated to prevent further transmission and provided with necessary medical treatment. In other words, contact tracing is a regulatory step identifying the risk of virus transmission, testing is a way of setting up targets of pharmaceutical intervention, and isolation and medical treatment 
is a means to fix the problem. The feasible goal of NPIs may not be obtaining a 'zero confirmed case,' but reducing the number of confirmed cases to a level that the health system can handle.

Braithwaite pointed out how easily a powerful regulator can be seduced to use coercive options when facing an imminent threat ${ }^{17}$. Finding a balanced way of harnessing State emergency power that effectively deters the spread of infection while minimally restraining human rights should be a goal of any democratic government. This is especially so because NPIs are anticipated to affect our everyday life continuously until vaccines and proper medicines are developed. Moreover, any personal information obtained in the process of contact tracing should be handled only for the sole, intended purposes, guaranteeing transparency of the process, and observing domestic and international human rights laws.

Of course, this paper has limitations. One of the issues is the reliability of the data. Especially the number of confirmed cases may not represent the actual number of infected cases. It is mainly dependent upon a government's testing policy or testing capacity. Besides, this paper considered a limited number of NPIs that have an impact on three types of human rights. However, a society's capacity to fight off the pandemic can be swayed by many factors, such as the quality of the public health care system, government's risk management capacity and agility, transparent flow of information, political leadership, compliance of businesses and citizens, and many more. Therefore, more extensive studies need to follow that examines what determines the success and failure of containing the COVID-19 pandemic.

\section{References}

$1 \quad$ Foucault, M. Discipline and punish: The birth of the prison. (Vintage, 2012).

2 Kaye, D. Disease pandemics and the freedom of opinion and expression. (A/HRC/44/49, para 5457, 2020).

3 Abouk, R. \& Heydari, B. The immediate effect of COVID-19 policies on social distancing behavior in the united states. Available at SSRN (2020).

4 Ferguson, N. et al. Report 9: Impact of non-pharmaceutical interventions (NPIs) to reduce COVID19 mortality and healthcare demand. (2020).

5 Flaxman, S. et al. Estimating the effects of non-pharmaceutical interventions on COVID-19 in Europe. Nature, 1-8 (2020).

6 Lai, S. et al. Effect of non-pharmaceutical interventions for containing the COVID-19 outbreak in China. Nature, doi:https://doi.org/10.1038/s41586-020-2293-x (2020).

7 Ryu, S., Ali, S., Jang, C., Kim, B. \& Cowling, B. Effect of Nonpharmaceutical Interventions on Transmission of Severe Acute Respiratory Syndrome Coronavirus 2, South Korea, 2020. Emerging Infectious Diseases 26 (2020).

8 Hale, T., Petherick, A., Phillips, T. \& Webster, S. Variation in government responses to COVID19. (Blavatnik School of Government, University of Oxford, Oxford, 2020).

9 van Rooij, B. et al. Compliance with COVID-19 Mitigation Measures in the United States (2020).

10 Hsiang, S. et al. The effect of large-scale anti-contagion policies on the COVID-19 pandemic. Nature, 1-9 (2020). 
11 Naude, J. et al. Worldwide Effectiveness of Various Non-Pharmaceutical Intervention Control Strategies on the Global COVID-19 Pandemic: A Linearised Control Model. medRxiv (2020).

12 Herren, C. M., Brownwright, T. K., Liu, E. Y., El Amiri, N. \& Majumder, M. S. Democracy and Mobility: A Preliminary Analysis of Global Adherence to Non-Pharmaceutical Interventions for COVID-19. Available at SSRN: http://dx.doi.org/10.2139/ssrn.3570206 (2020).

13 Corbet, S. \& Chan, K. in Voice of America News (2020).

14 Zastrow, M. in Nature (2020).

15 Workman, J. The proportion of COVID-19 cases that are asymptomatic in South Korea: Comment on Nishiura et al. International Journal of Infectious Diseases 96, 398 (2020).

16 Nishiura, H. et al. Estimation of the asymptomatic ratio of novel coronavirus infections (COVID19). International Journal of Infectious Diseases 94, 154 (2020).

17 Braithwaite, J. Limits on Violence; Limits on Responsive Regulatory Theory. Law \& Policy 36, 432-456, doi:10.1111/lapo.12026 (2014). 


\section{Methods}

Decrease rate of increase in cumulative COVID-19 confirmed cases

To define the decrease rate of increase in the cumulative confirmed cases (DRICs), we first define $\bar{y}_{t}$ as the mean of the cumulative confirmed cases for three days to make the cumulative confirmed case curve smooth as below.

$$
\bar{y}_{t}=\frac{y_{t-1}+y_{t}+y_{t+1}}{3}
$$

Where,

$\mathrm{y}_{\mathrm{t}}=$ cumulative confirmed cases of COVID-19 on day $\mathrm{t}$; and

$\mathrm{t}=$ number of days from the 1st of January, 2020.

Second, we define $a_{t}$ as the average rate of increase in confirmed cases over a weak (see Figure 2).

$$
a_{t}=\frac{\Delta \bar{y}}{\Delta t}=\frac{\bar{y}_{t+3}-\bar{y}_{t-3}}{6}
$$

Finally, we define DRICs $(\Phi)$ as below (see Figure 2).

$$
\Phi=\frac{a_{t^{\prime}}}{a_{t^{\prime}+6}}
$$

Where

$\mathrm{t}^{\prime}=$ the day $(\mathrm{t})$ when $\mathrm{a}_{\mathrm{t}}{ }^{\prime}=\max \left(\mathrm{a}_{\mathrm{t}}\right)$. 


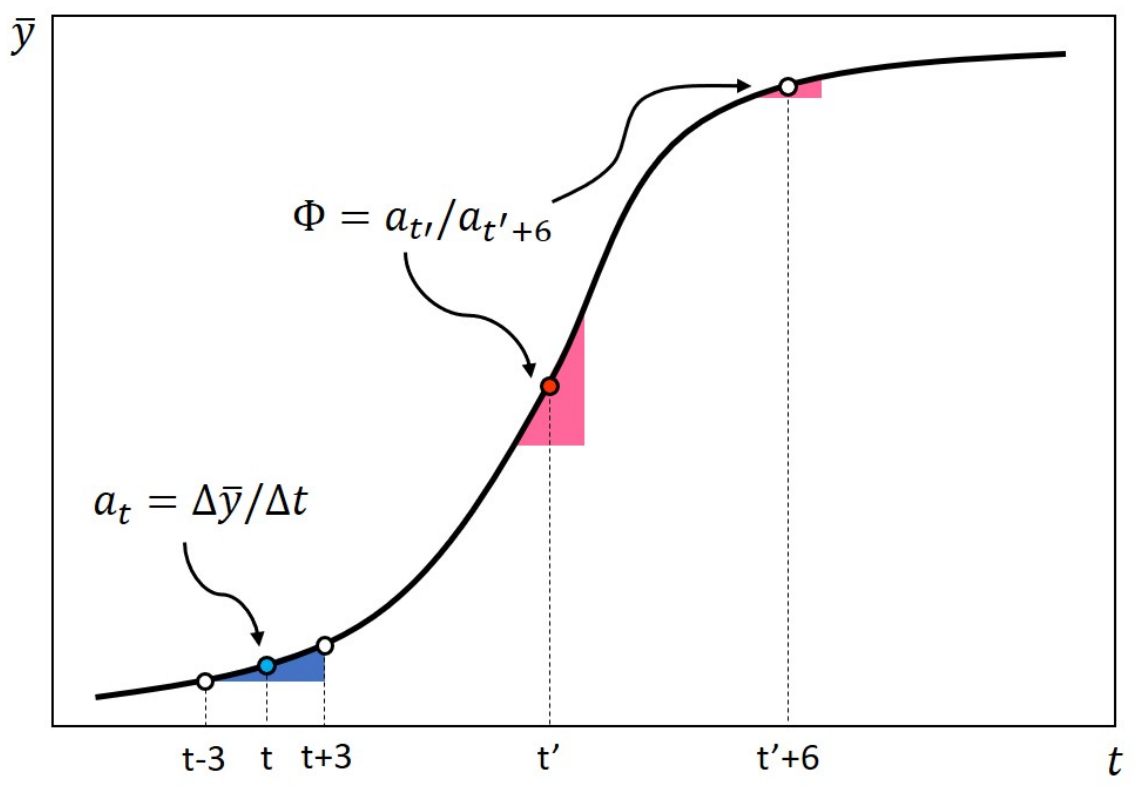

$<$ Figure $2>$ Graphical definition of $\mathrm{a}_{\mathrm{t}}$ and $\Phi$

\section{Standardized Non-Pharmaceutical Interventions}

We basically standardized NPIs in the same way as Hale et al. ${ }^{8}$ considering description 1 (degree) and description 2 (targeted or general) and the process is as below.

$$
I_{j, t \prime}=\frac{v_{j, t}-0.5(\text { only if description } 2=\text { targeted })}{\max \left(v_{j}\right)}
$$

Where,

$\mathrm{I}=$ standardized NPI score;

$\mathrm{j}=$ kind of NPIs (A1 to A4, M1 to M4, and P1);

$\mathrm{v}_{\mathrm{j}, \mathrm{t}^{\prime}}=$ the value of description 1 of NPI $\mathrm{j}$ on day $\mathrm{t}^{\prime}$;

For graph clarity of the ten countries, we combine NPIs into the ones limiting assembly, movement, and privacy as below.

$$
\begin{gathered}
I_{\bar{A}, t}=\frac{1}{4}\left(I_{A 1, t}+I_{A 2, t}+I_{A 3, t}+I_{A 4, t}\right) \\
I_{\bar{M}, t}=\frac{1}{4}\left(I_{M 1, t}+I_{M 2, t}+I_{M 3, t}+I_{M 4, t}\right) \\
I_{\bar{P}, t}=I_{P 1, t}
\end{gathered}
$$




\section{Data availability}

Oxford COVID-19 Government Response Tracker (OxCGRT) database we used for confirmed case counts for the 108 countries and changes in their NPIs for the time period in our study is available at https://www.bsg.ox.ac.uk/research/research-projects/coronavirus-governmentresponse-tracker\#data.

\section{Code availability}

All source code and data necessary for the replication of our results and figures is available at https://github.com/hahwang0309/covid19-humanRights 
a. United Kingdom

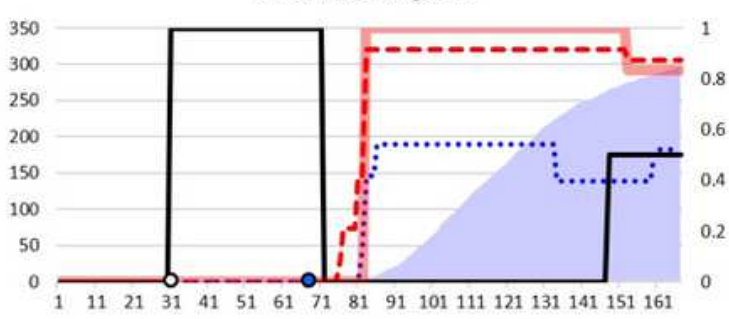

b. United States

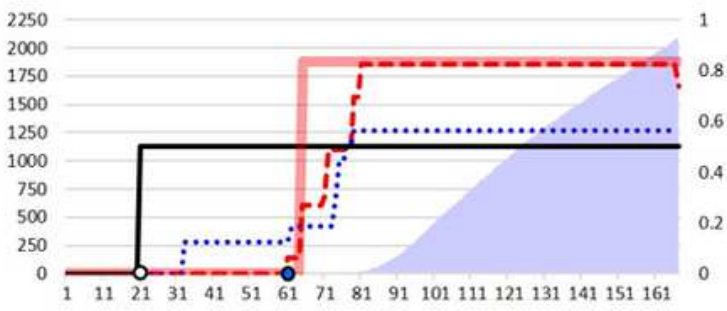

c. Sweden

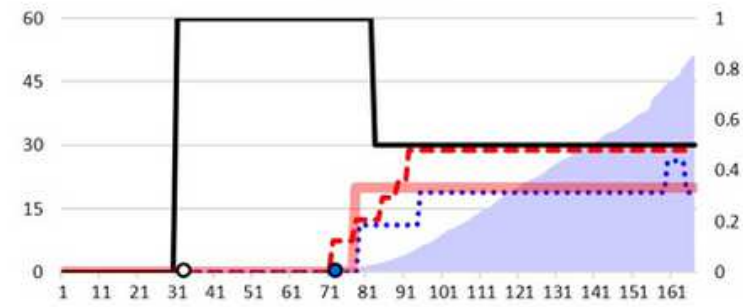

d. France

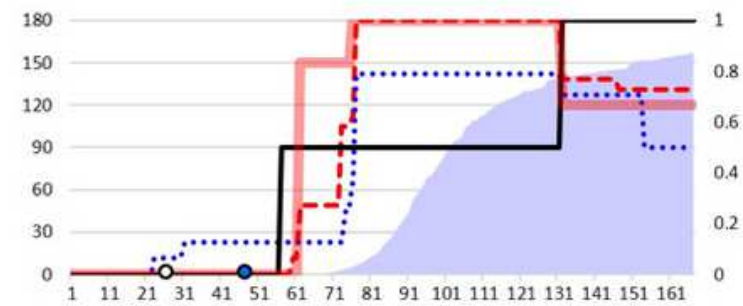

e. Slovenia

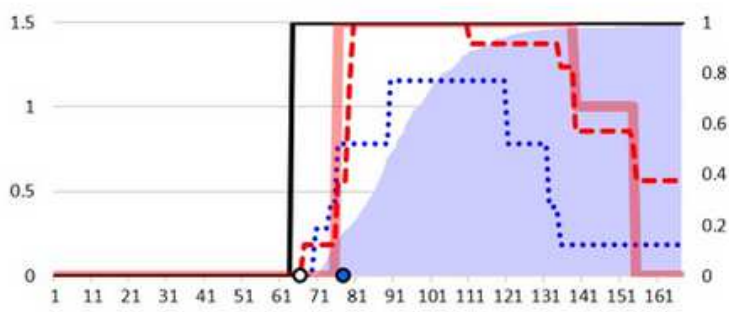

Cumulative number of confirmed cases
- $1^{\text {st }}$ confirmed case

..... Movement restriction f. China

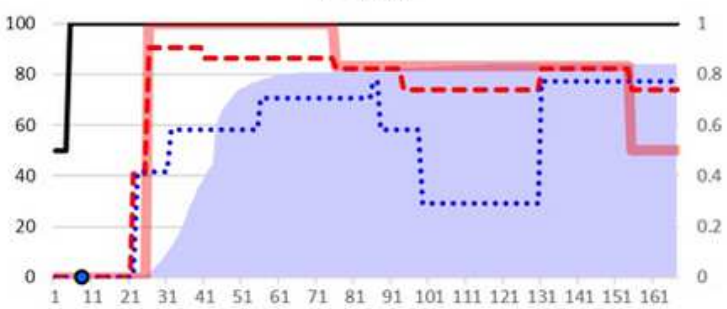

g. South Korea

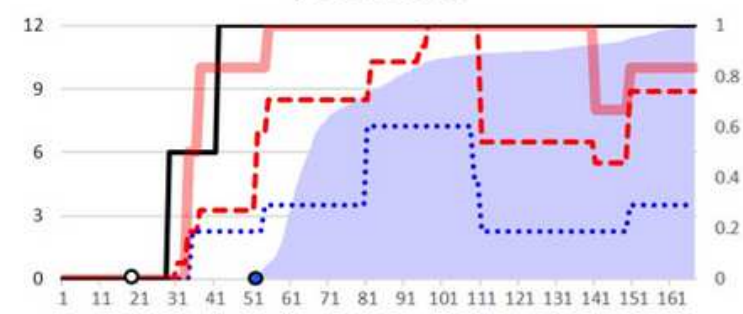

h. Japan

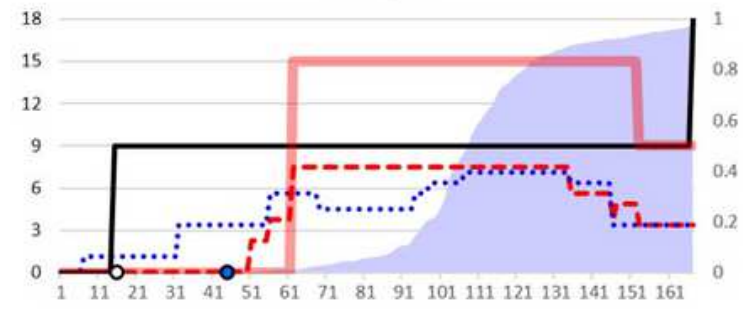

i. Vietnam

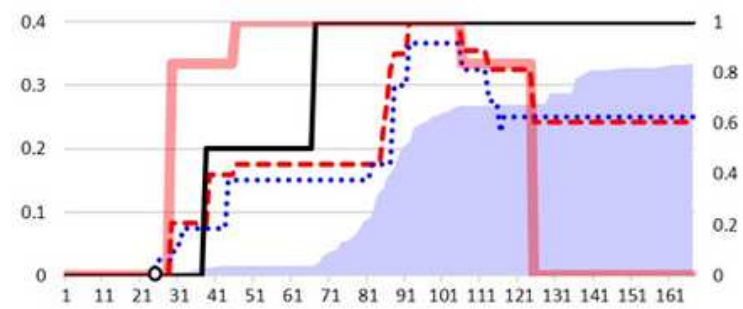

j. New Zealand

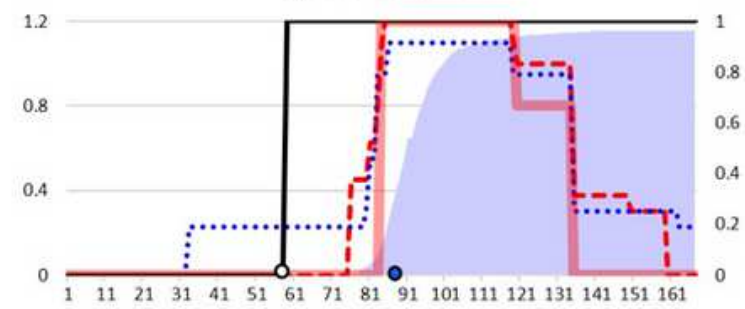

- $1^{\text {st }}$ death

Privacy restriction

\section{Figure 1}

Changes in NPIs and cumulated number of confirmed cases of COVID-19 of the selected countries. Note: The horizontal axis denotes the number of days from the 1st of January, 2020. The right vertical axis 
denotes the standardized score of NPIs. The left vertical axis denotes the number of confirmed cases of COVID-19, and the unit of confirmed cases is a thousand people.

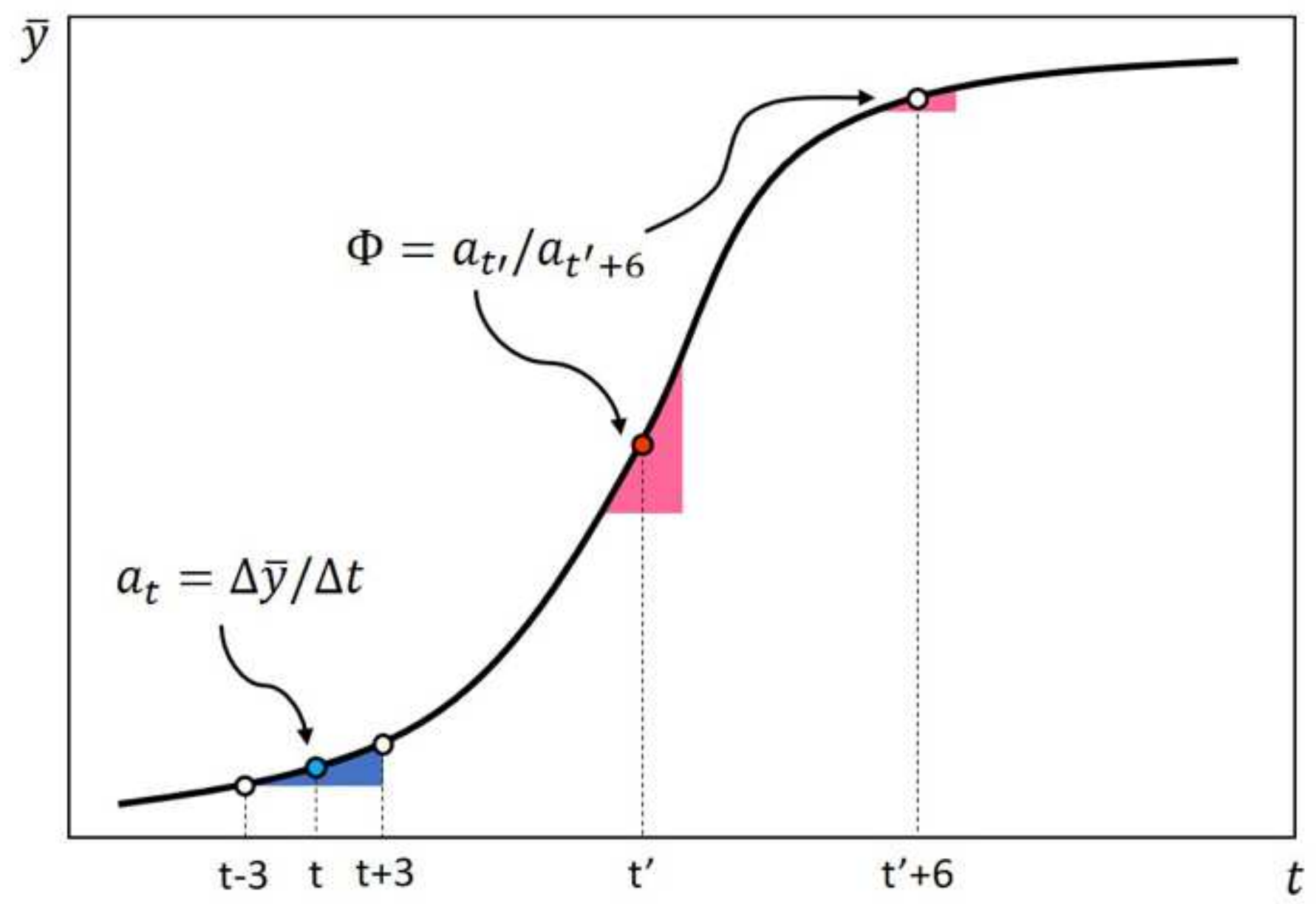

Figure 2

Graphical definition of at and $\Phi$ 